\title{
LA ASOCIACIÓN ANDALUZA DE SEMIÓTICA
}

\section{Manuel Ángel Vázquez Medel}

Presidente de la Asociación Andaluza de Semiótica

\section{INTRODUCCIÓN}

Sin lugar a dudas, lo más importante de una asociación científica en el ámbito de las Ciencias Sociales y las Humanidades, como la Asociación Andaluza de Semiótica, son sus aportaciones investigadoras y la capacidad para difundir determinadas sensibilidades en diversos ámbitos profesionales y docentes. Ambos objetivos han ido determinando la organización de Simposios, Seminarios introductorios y avanzados, Cursos y publicaciones. En Andalucía, la Semiótica nunca ha sido contemplada doctrinal ni dogmáticamente, aunque la atención a los diversos movimientos y tendencias ha sido constante, al igual que la voluntad de rigor científico en los trabajos. Pero siempre sin exclusiones, entendiendo la Semiótica como una Teoría Crítica de la Cultura (y una praxis de análisis y creación); como un saber para la vida, más allá de huecas especulaciones.

Globalmente, podría caracterizarse la investigación semiótica en Andalucía - dentro de una extraordinaria pluralidad de fundamentos 
teóricos y metodológicos y ámbitos de aplicación - como un campo de trabajo interdisciplinar, cada vez más volcado hacia la comunicación intercultural, en el que la superación del inmanentismo estructuralista ofrece peculiares perfiles de interés por la pragmática, la hermenéutica, la dimensión socio-histórica y material de la semiosis, e incluso por la poética del (y de lo) imaginario.

Aunque en algún caso con carácter incipiente y emergente, comienzan a dibujarse ciertas tendencias en la investigación semiótica andaluza: los investigadores de Granada ocupan un lugar de primer orden en el ámbito de la Semiótica social, de la sociocrítica, y la Sociología de la Literatura, dirigida por Antonio Sánchez Trigueros (Madrid: Síntesis, 1997) es ya un manual de obligada referencia en su ámbito. Ello no impide en otras aproximaciones el mayor énfasis sobre aspectos de la historiografía y la formulación de los saberes literarios (A. Chicharro, G. Pulido en la Universidad de Jaén), sobre la semiótica de la cultura lotmaniana (M. Cáceres), la poética bajtiniana (D. Sánchez Mesa), la atención por la psicocrítica y la crítica feminista (C. Martínez Romero), la hermenéutica (S. Wahnon), la semiótica teatral (M. A. Grandes, M. J. Sánchez Montes) o interesantísimas aplicaciones a la métrica (A. Carvajal) o a la narrativa picaresca (F. Linares).

El interés por la semiótica teatral, ámbito en el que hay que citar las aportaciones pioneras de Jorge Urrutia, está también presente en diversos trabajos que encuentran como marco la Universidad de Córdoba (P. Moraleda, A. Sánchez), así como en la investigación de F. Cantalapiedra.

Aunque en toda Andalucía se presta una especial atención a la narratología, diversos grupos han llegado a adquirir cierta consistencia propia. Así, en la Universidad de Almería, bajo la coordinación de J. Valles, el Grupo de Investigación «Teoría de la Literatura y Literatura Comparada» ha ofrecido varios volúmenes de interés (M.I. Navas, J. Heras, F. Álamos). En Sevilla, el Curso Superior de Narratología, coordinado por $\mathrm{C}$. Pérez, supone la consolidación de una plataforma en la que participan, especialmente, el Grupo Temático-Estructural y el Grupo de Investigación en Teoría y Tecnología de la Comunicación. Hemos de mencionar también los Simposios Internacionales sobre Narrativa Hispánica Contemporánea, organizados por la Fundación Luis Goytisolo, que reúnen en El Puerto de Santa María (Cádiz) cada noviembre a los más destacados creadores, teóricos y críticos, si bien sin explícitas orientaciones semióticas. 
En la Universidad de Cádiz, algo más alejados de una investigación estrictamente semiótica, hay que destacar notables esfuerzos en el ámbito de la Retórica (J. A. Hernández Guerrero, Pérez Castellano), así como en la investigación sobre literatura contemporánea (M. Ramos Ortega, R. Martínez Galán, A. S. Pérez Bustamante, A. González Troyano).

La joven Universidad de Huelva comienza a incorporarse con entusiasmo a la investigación semiótica, fundamentalmente en el ámbito del comparatismo (A. Ramírez de Verger, M. A. Márquez, L. Gómez Canseco) y de la investigación literaria, estética y artística (J. Prados, E. Navarro). En mayo de 1998 se han celebrado unas importantes Jornadas, Orientaciones actuales en Semiótica, preparatorias del Simposio de 1999, que pronto serán publicadas.

La aportación más destacada de los investigadores de la Universidad de Sevilla ha sido la formulación de una Semiótica Transdiscursiva (cf. M. Á. Vázquez Medel, Introducción a la Semiótica Transdiscursiva, Sevilla: Cuadernos de Comunicación, 1998), o semiótica de la transcendencia discursiva, que radicaliza ciertas investigaciones sobre fenómenos intertextuales en las últimas décadas, incorporando sensibilidades provenientes de la hermenéutica y de la nueva teoría de la comunicación y de la cultura, y que ha sido aplicada eficazmente a objetos muy diversos: Teoría de la Comunicación (A. Acosta), Semiótica de la publicidad (J. Rey), Semiótica de las ideologías (A. Huici, S. Carazo), Análisis fílmico (C. Colón), etc. La proyección comunicacional, introducida por Jorge Urrutia (cf. su Literatura y Comunicación, Madrid: Biblioteca Nueva, 1997), conoce en estos momentos interesantes aplicaciones a la teoría general del iconismo (I. Rodríguez Cunill), a las relaciones entre cine y literatura (E. Civila de Lara, A. Recio), periodismo y literatura (P. Bellido), a la indagación en el ámbito de la caología (I. Roldán) o de la infografía (F. Contreras). Más recientes son otras líneas de trabajo más específicas, como la semiótica musical (F. J. Cuadrado).

La aplicación de la teoría literaria y de la semiótica a la enseñanza de la literatura tiene una destacadísima representación en los trabajos de E. Barroso o los realizados bajo su dirección (J. Corriente).

Sin duda, la vitalidad con que en el sur de España se desarrollan investigaciones de impronta semiótica impide elaborar un mapa exhaustivo. Una completa referencia de muchos de los trabajos aquí aludidos puede consultarse en los diversos elencos bibliográficos de J. Romera, 
cuya presencia, unida a la de J. Urrutia y J. Talens, ha sido fundamental en el desarrollo de la semiótica en Andalucía.

\section{HITOS ORGANIZATIVOS}

A pesar de algunos antecedentes aislados, el interés por la semiótica en Andalucía remonta al inicio de la década de los setenta. Podemos encontrar, en varias de sus Universidades —especialmente Sevilla y Granada-, diversas aportaciones de orientación semiótica, implícita o explícita, especialmente en el ámbito de la Teoría de la Literatura. Igualmente, algunos profesores andaluces, que comienzan a desarrollar su actividad docente e investigadora en otras Universidades españolas van a tener un papel muy destacado en el desarrollo de la semiótica en España.

La presencia de Andalucía en el Congreso Internacional sobre Semiótica e Hispanismo, organizado en 1983, en el CSIC, por Miguel Ángel Garrido Gallardo -que había sido Catedrático de Teoría de la Literatura en la Universidad de Sevilla - fue, sin duda, notable. Sería, precisamente, el granadino José Romera Castillo - fundador también de la AAS - quien propusiera, en este marco, la creación de la Asociación Española de Semiótica (AES). A Garrido, primer presidente de la AES, sucedería en el cargo Alicia Yllera, quien también había sido Catedrática de Filología Francesa de la Universidad de Sevilla y, más tarde, José Romera. Las relaciones con la AES, hasta el momento actual, bajo la presidencia de José M. ${ }^{a}$ Pozuelo Yvancos, son inmejorables.

La investigación semiótica en Andalucía tiene, en sus inicios, dos claros impulsores: el profesor Jorge Urrutia Gómez quien, desde su incorporación a la Universidad de Sevilla, en 1979, dinamizará la actividad investigadora —orientándola hacia el ámbito comunicacionalasí como la dimensión organizativa, y el profesor Antonio Sánchez Trigueros, quien desarrollará una intensa actividad en el marco de la Teoría de la Literatura y la Sociosemiótica, desde Granada.

Un importante punto de partida en las actividades sobre Semiótica en Andalucía fue el Ciclo sobre «Cine y Semiología», celebrado del 13 al 19 de octubre de 1980, en el marco del I Festival Internacional de Cine de Sevilla. Dirigido por Manuel Ángel Vázquez Medel, entonces 
Coordinador General de Redacción de la Enciclopedia de Andalucía, contó con la participación de Luis Guembe («Crítica cinematográfica y semiótica»), Roger Odin («Análisis de Le tempestaire de Epstein») y Jorge Urrutia («El lenguaje cinematográfico: construcción y destrucción de un concepto»). Los encuentros en la Universidad Antonio Machado de Baeza, impulsados por Sánchez Trigueros con la colaboración de Chicharro, marcaron momentos importantes en la investigación semiótica y literaria.

La presentación oficial de la Asociación Andaluza de Semiótica tuvo lugar el martes, 20 de mayo de 1986, en el Aula Magna de la Facultad de Filología de Sevilla, a cargo de Jorge Urrutia y Manuel Ángel Vázquez Medel. Walter Mignolo pronunció a continuación una conferencia sobre «Gramática, convenciones y normas (reflexión sobre la ficción, la literatura y la historia)».

La primera Asamblea General de la AAS se celebraría el 9 de julio de 1986, en el marco del «Seminario sobre Antropología Semiótica» del VII Curso de Verano de San Roque, dirigido por Jorge Urrutia, y en el que participaron, además de su director, Jenaro Talens, Manuel A. Vázquez Medel, Francesco Casetti y J. Luis Caramés Lage. La primera Junta Directiva estuvo integrada por:

Presidente: Jorge Urrutia (Univ. de Sevilla).

Vicepresidente: Antonio Sánchez Trigueros (Univ. de Granada).

Director de Discurso: Manuel Ángel Vázquez Medel (Univ. de Sevilla).

Tesorera-Secretaria: Pilar Bellido Navarro (Univ. de Sevilla). Más tarde sería sustituida por Isabel Román Gutiérrez (Univ. de Sevilla)

Vocales: Pilar Moraleda (Univ. de Córdoba).

Rosario Martínez Galán (Univ. de Cádiz).

Carmen Martínez Romero (Col. Univ. de Almería).

Begoña López Bueno (Univ. de Sevilla).

Jorge Urrutia sería Presidente de la AAS hasta 1991, momento en el que Antonio Sánchez Trigueros fue elegido Presidente, en el IV Simposio Internacional de Córdoba, en el que también se nombraba a Urrutia Presidente de Honor. Francisco Linares Alés fue Secretario- 
Tesorero en esta etapa, sin que se produjeran otros cambios en la Directiva.

Manuel Ángel Vázquez Medel fue elegido Presidente en la Asamblea celebrada en Sevilla en octubre de 1996, pasando a ser Director de Discurso Ángel Acosta Romero, quien hasta dicho momento había sido Secretario de Redacción. En la actualidad, la composición de la Junta Directiva es la siguiente:

Presidentes de Honor: Jorge Urrutia (Univ. Carlos III) y Antonio Sánchez Trigueros (Univ. de Granada).

Presidente: Manuel Ángel Vázquez Medel (Univ. de Sevilla)

Vicepresidente: Antonio Chicharro Chamorro (Univ. de Granada).

Director de Discurso: Ángel Acosta Romero (Univ. de Sevilla).

Tesorera-Secretaria: Yolanda Rodríguez - Mercedes Arriaga (Univ. de Sevilla).

Vocales: Ascensión Sánchez (Univ. de Córdoba).

Carlos Fernández Serrato (Cádiz).

Francisco Álamo Felices (Univ. de Almería).

Genara Pulido Tirado (Univ. de Jaén).

José Antonio García - Josefina Prados (Huelva).

\section{LOS SIMPOSIOS INTERNACIONALES}

La AAS, que desde su fundación contó con más de un centenar de Profesores Universitarios, de Instituto y Profesionales, y que en la actualidad cuenta con casi quinientos asociados, cifraría sus dos objetivos básicos en la celebración de Simposios Internacionales anuales - a partir de 1990, bienales, para alternar con los de la AES- y en la publicación de Discurso. Revista Internacional de Semiótica y Teoría Literaria. 
El I Simposio Internacional se celebró en diciembre de 1987 en Almería. En él se presentó el primer número de la revista Discurso.

El II Simposio Internacional se celebró en septiembre de 1988 en Cádiz. Existe una pre-publicación con los resúmenes de las ponencias y comunicaciones (M. Á. Vázquez Medel (ed.) (1988): II Simposio Internacional de la AAS. Abstracts. AAS, Cádiz).

El III Simposio Internacional se celebró del 14 al 16 de diciembre de 1989 en Granada. Supuso un importante paso adelante en la participación de ponentes y comunicantes. Sus trabajos se editaron en microfichas (A. Sánchez Trigueros - A. Chicharro Chamorro (1990): Actas del III Simposio Internacional de la AAS. Serv. de Publ. de la Univ. de Granada).

El IV Simposio Internacional de la AES se celebró en 1990 en Sevilla. Las actas se publicaron en dos volúmenes [AES (ed.) (1992): Investigaciones Semióticas IV. Descubrir, Inventar, Transcribir el Mundo, Visor Libros, Madrid]. La AAS aceptó celebrar, a partir de entonces, con carácter bienal, sus Simposios.

El IV Simposio Internacional de la AAS se celebró en diciembre de 1991 en Córdoba. Además de la edición en microfichas (P. Moraleda - A. Sánchez (eds.) (1992): Actas del IV Simposio Internacional de la $A A S$. Serv. de Publ. de la Univ. de Córdoba), las ponencias se recogieron en un pequeño volumen impreso.

El V Simposio Internacional se celebró en diciembre de 1993 en Almería (J. Valles (ed.) (1995): Actas del V Simposio Internacional de la Asociación Andaluza de Semiótica. Universidad de Almería).

El VI Simposio Internacional se celebró en octubre de 1996 en Sevilla, como Congreso Extraordinario Conmemorativo del $10{ }^{\circ}$ Aniversario de la AAS, con la participación de las figuras más destacadas de la investigación semiótica en el ámbito hispánico (cf. M. Á. Vázquez Medel - A. Acosta (eds.) (1998): La semiótica actual. Actas del VI Simposio Internacional de la AAS, Sevilla: Alfar). Al coincidir este mismo año el simposio AAS con el de la AES en Zaragoza, y a fin de volver a la alternancia bienal, se hizo el esfuerzo extraordinario de convocar el VII Simposio Internacional en septiembre de 1997 en Baeza, dirigido por Antonio Chicharro, con el tema Espacios artísticos - Espacios Literarios, cuyas actas se encuentran en prensa.

Está previsto celebrar el VIII Simposio Internacional de la Asociación Española de Semiótica en diciembre de 1998 en Granada, con 
el tema Miradas y voces de fin de siglo, siendo la primera ocasión que una Comunidad del Estado Español acoge por segunda vez un Simposio de AES.

El VIII Simposio Internacional de la AAS se celebrará en La Rábida (Huelva) en 1999 y, coincidiendo con el Simposio de la Asociación Latinoamericana de Semiótica de La Coruña, tendrá un marcado carácter latinoamericano. El Simposio del año 2001 se celebrará en Málaga, con lo que las ocho provincias de Andalucía habrán participado en esta primera fase como sedes de los encuentros de AAS.

\section{EL SIMPOSIO IN MEMORIAM IURI M. LOTMAN}

Lugar aparte, entre los encuentros de la AAS, merece el Simposio dirigido por Manuel Cáceres del 26 al 28 de octubre de 1995 en la Universidad de Granada in memoriam Iuri M. Lotman, auspiciado también por la IASS-AIS y la AES. Participaron en sus Sesiones Plenarias Igor Chernov, Mijail Lotman, Desiderio Navarro, Cesare Segre, Peeter Torov y Boris Uspenski, y actuaron como ponentes José Domínguez Caparrós, Miguel Ángel Garrido Gallardo, Jorge Lozano, José María Pozuelo Yvancos, Antonio Sánchez Trigueros, Jenaro Talens, Jüri Talvet, Jorge Urrutia y Manuel Á. Vázquez Medel. Un numeroso y destacadísimo grupo de profesores ofrecieron comunicaciones agrupadas en sesiones sobre Semiótica General, Lingüística, Teoría del Texto, Literatura, Cultura, Artes no verbales (Artes visuales, música, cine), Semiótica y Teoría de la Literatura del siglo XX. Una selección de estos trabajos aparece en el volumen de M. Cáceres (ed.) (1997): En la esfera semiótica lotmaniana. Estudios en honor de Iuri Mijáilovich Lotman, Valencia: Episteme.

Como se indica en la nota introductoria a Discurso 8, el primer volumen de una revista internacional publicado in memoriam I. Lotman, la Semiótica de la Cultura de la escuela de Tartu-Moscú ha tenido en los investigadores de Andalucía una temprana e intensa acogida, así como la influencia del pensamiento bajtiniano (cf. los trabajos de Domingo Sánchez Mesa). Una destacada investigadora sobre Mijail Bajtín, Iris M. Zavala, ha sido en numerosas ocasiones invitada de honor en las Universidades de Granada y Sevilla. 


\section{LOS CURSOS DE INTRODUCCIÓN Y AVANZADOS}

San Roque (Cádiz) fue, durante varios años, un punto de referencia obligado en la geografía semiótica española. El impulso de la investigación semiológica se unía así al prestigio alcanzado por los Cursos de Verano, dirigidos - sobre diversas dimensiones psicológicas y psiquiátricas de interés social - en dicha localidad por Carlos Castilla del Pino. Coordinados en todas sus convocatorias por Jorge Urrutia, además de proporcionar el marco fundacional de la AAS en 1986 («Seminario de Antropología Semiótica»), en 1987 se celebró un Seminario dedicado a los fenómenos de transcodificación, en el que participaron, además de su director, Elena Dagrada, François Jost, Jenaro Talens, Vicente Sánchez Biosca y M. Á. Vázquez Medel.

En 1988, el Seminario de Semiótica estuvo dedicado al estudio de los clásicos españoles, y contó, además de las ponencias-marco —ofrecidas por Urrutia y Vázquez Medel - con la participación de $\mathbf{M}$. Catherina Ruta, E. Canceliere, José Romera Castillo y César Oliva. Julio de 1989 acogió por última vez este Seminario de Semiótica, dedicado a textos españoles contemporáneos, que contó con la participación de Rosa de Diego, Miguel Á. Garrido Gallardo, Juan Montero, Pilar Moraleda, J. Urrutia y M. Á. Vázquez Medel.

En el mes de enero de 1987 la AAS, en colaboración con la Universidad de Córdoba, organizó un Seminario de Introducción a la Semiótica con la participación de los profesores Moraleda, Talens, Urrutia y Vázquez Medel. A partir de entonces se han organizado varios ciclos en Córdoba, el último de los cuales se celebró en 1995.

En las sesiones de verano de UNALMA (1987), celebradas en Asilah (Marruecos), colaboró la AAS en el Seminario sobre Forma y sentido de la arquitectura morisca.

La Universidad de Granada, desde su Área de Teoría de la Literatura, y en colaboración con la AAS, ha ofrecido con periodicidad casi anual un ciclo de Primavera sobre cuestiones actuales de Teoría Literaria y Semiótica. El último de ellos se celebró en mayo de 1997 con el título Signos \& Sociedad (Introducción a la Semiótica).

Durante el curso 1987/88 la Asociación Andaluza de Semiótica organizó, en colaboración con el ICE y la Facultad de Filología de la Universidad de Sevilla, el Seminario Permanente Sistemas de Análisis de textos artísticos, que contó con la participación de Carlos Reis, 
Luciano García Lorenzo, Jenaro Talens, Claudio Guillén y Sandra Melloni.

En mayo de 1995 se celebró en la Facultad de Ciencias de la Información de la Universidad de Sevilla un encuentro con el tema «Cuestiones actuales en Semiótica y Teoría de la Comunicación», que reunió a José Antonio Mingolarra, Santos Zunzunegui y José Manuel Pérez Tornero.

La AAS también ha colaborado en varios Cursos promovidos por la Consejería de Educación y Ciencia de la Junta de Andalucía, como las Ponencias «Introducción a la Semiótica» y «Pragmática, Semiótica y Comunicación», ofrecidas en los Cursos de actualización científicodidáctica celebrados en Torremolinos (Málaga) en 1992, 1993 y 1994, o el Curso «Semiótica y su aplicación en el Aula. Enfoque comunicativo» (1995).

Durante los cursos 1995/96, 96/97 y 97/98 la Universidad de Huelva ha organizado un Seminario sobre Semiótica y Estética, impartido por M. Á. Vázquez Medel en el marco del Curso de Experto «La enseñanza de las Artes Plásticas y Visuales en la Educación».

\section{LOS SEMINARIOS DE NARRATOLOGÍA}

A partir de 1990, la AAS, en colaboración con la Universidad de Sevilla (especialmente el Departamento de Filología Francesa y los Grupos de Investigación Temático Estructural y de Teoría y Tecnología de la Comunicación), ha venido celebrando un Seminario de Narratología que, en su primera convocatoria, contó con Javier del Prado y Manuel Á. Vázquez Medel, quienes, desde entonces, han venido participando en todas las convocatorias sucesivas. Coordinados por Concepción Pérez, tras la reflexión inicial sobre diversos problemas de la Narratividad, dedicó su II convocatoria al estudio De la narratividad a la Poeticidad; la III convocatoria (1992) tuvo como tema Narratividad. Poeticidad. Discursividad. En 1993 en el IV Seminario de Narratología se estudió La literatura en sus límites. Género y transgresión, tema que se retomó en 1995 - tras el paréntesis del Coloquio Internacional de Niza - como Curso Superior de Narratología. El género literario, y que fue publicado en un volumen por la Universidad de Sevilla, bajo la coordinación de Concepción Pérez. 
Como se ha indicado, en 1994 se celebró en Niza el $l$ Coloquio Internacional de Narratología, en colaboración del Centro de Narratología aplicada de la Universidad de Sophia-Antipolis con la Universidad de Sevilla. El tema: Le personnage romanesque. El coloquio internacional estuvo presidido por los profesores Mieke Bal y Manuel Ángel Vázquez Medel (cf. G. Lavergne (ed.) (1995): Colloque International «Le personnage romanesque», Cah. de Narratologie, 6, Univ. de Nice). El II Coloquio Internacional de Narratología, celebrado en la Universidad de Sevilla en 1997 se dedicó al estudio de las relaciones entre Creación espacial y narración literaria. Sus Actas serán publicadas por la Universidad de Sevilla.

Un lugar destacado ha tenido, desde sus orígenes, la investigación narratológica en la Universidad de Almería, bajo la coordinación de José Valles Calatrava. En 1996, y con estrecha colaboración de la AAS, el Grupo de Investigación «Teoría de la Literatura y Literatura Comparada» organizó en Almería el I Curso de Teoría de la Narrativa, con la participación de A. Sánchez Trigueros, José R. Valles Calatrava, M. Á. Vázquez Medel, J. Domínguez Caparrós, J.M. Pozuelo Yvancos, J. Talens, J. Heras, J. Heredia Maya, M.I. Navas Ocaña y F. Álamos.

Igualmente en la Universidad de Almería hay que citar los importantes encuentros sobre Literatura Comparada bajo la dirección de Emilio Barón.

\section{Encuentros sobre Poética del (y de lo) Imaginario}

Del 7 al 11 de mayo de 1990, en la -entonces- recientemente creada Facultad de Ciencias de la Información de la Universidad de Sevilla se celebró, impulsado por la AAS, un importante Seminario Internacional sobre Poética de lo imaginario, que reunió en un debate fecundo a investigadores de la esfera semiótica con especialistas en Poética del (y de lo) Imaginario y Mitocrítica, especialmente vinculados al Círculo de Eranos. El 5 de mayo El Correo de Andalucía dedicó un suplemento monográfico al encuentro, en el que participaron Simone Vierne (portadora también del saludo de Gilbert Durand), Fátima Gutiérrez, Alicia Yllera, Adrián Huici, Marta Segarra, Lola Bermúdez, Ángel Acosta, Carmen Ramírez, Jorge Urrutia, Andrés 
Ortiz Osés, Alain Verjat, Rosa de Diego, Javier del Prado, Jenaro Talens y su director, M. Á. Vázquez Medel.

El II Simposio Internacional sobre lo Imaginario se celebró en 1992 en el Foro Iberoamericano y en la Universidad de Santa María de La Rábida (Huelva). Su tema monográfico, El cuerpo y el estudio de sus representaciones, reunió a destacados investigadores internacionales y de las Universidades españolas como Cesare Segre, Omar Calabrese, Jenaro Talens, Giulia Colaizzi, Alain Verjat, Andrés Ortiz Osés, Javier del Prado, Rosa de Diego, Chantal Maillard, Jesús Aguado, Lola Luna, Ismael Roldán, Manuel Á. Vázquez Medel, etc.

\section{LA RELACIÓN CON O'TRAS ASOCIACIONES E INSTITUCIONES}

Como habrá podido observarse, la relación de la AAS con otras asociaciones de Semiótica es muy intensa. Debemos destacar, especialmente, la relación con la AES y con la Asociación Vasca de Semiótica, en cuyas Jornadas Internacionales siempre ha habido una importante representación de investigadores de Andalucía. A partir de 1998 se han intensificado los contactos con Italia $\left(1 .{ }^{a}\right.$ sesión del Foro HispanoItaliano de Semiótica, en Sevilla, que contó, entre otros investigadores con la presencia de los profesores Augusto Ponzio y Susan Petrilli de la Universidad de Bari) y con Francia (Seminario de Sociocrítica, en Granada y Sevilla, con los profesores Edmond Cross y Annie Bussière, de la Universidad de Montpellier).

La AAS ofrece en Discurso traducciones e informes sobre la investigación semiótica en diferentes países (Italia, Francia, Canadá, Estonia, Rusia, etc.) y potencia el intercambio de sus publicaciones con otras revistas internacionales de Semiótica.

La AAS ha funcionado como lugar de encuentro de Grupos de Investigación, y su carácter interuniversitario salva del peligro de aislamiento que, en Ciencias Sociales y Humanidades, podría tener una mal entendida autonomía universitaria.

Especialmente estrechos son los lazos de la AAS con grupos de investigación sobre el imaginario y mitocrítica, pese a que muchos piensan que sus orientaciones son irreconciliables. Prueba de que ello 
no es así es la acogida en A. Ortiz-Osés- P. Lanceros (dirs.) (1997): Diccionario interdisciplinar de Hermenéutica, Bilbao: Univ. de Deusto, de un artículo dedicado a la noción de «Discurso» que recoge las orientaciones propias de la semiótica transdiscursiva.

\section{PUBLICACIONES}

Además de Discurso, la AAS mantiene, en colaboración con Ediciones Alfar (Sevilla), una Colección titulada Semiótica y Crítica, dirigida por M. Á. Vázquez Medel, con los siguientes títulos:

Urrutia, J. (1984): Imago Litterae. Cine y Literatura.

Chicharro Chamorro, A. (1987): Literatura y Saber.

Urrutia, J. (1990): Sistemas de Comunicación.

Zunzunegui, S. (1990): Metamorfosis de la mirada.

Rey, J. (1992): La significación publicitaria.

Chicharro Chamorro, A. (1993): Teoría, Crítica e Historia Literarias Españolas.

Vázquez Medel, M. Á.- Acosta, A. (1998): Charles Morris: Estética y Teoría de los signos.

Comenzada su publicación en 1996, con ocasión del Simposio Conmemorativo del $10 .^{\circ}$ aniversario, y relanzado en 1998 , Los signos en rotación es el Boletín informativo de la AAS, que complementa así, con datos operativos, la función científica de Discurso.

WEB-SITE

Desde 1995, el Grupo de investigación en Teoría y Tecnología de la Comunicación de la Universidad de Sevilla ofrece en su web-site 
(http://www.cica.es/aliens/gittcus) información actualizada sobre la AAS, así como la versión en internet del Boletín Los signos en rotación.

En la actualidad se está preparando un CD-Rom con los textos de Discurso y las Actas de los diversos encuentros AAS, que esperamos esté disponible en 1999.

Un logro importante de la AAS en los últimos años ha sido impulsar la presencia de asignaturas de Semiótica en diversos planes de estudio de Ciencias Sociales y Humanidades, como en la Licenciatura de Teoría de la Literatura de la Universidad de Granada, o las cinco asignaturas de Semiótica (Semiótica de la comunicación, Semiótica de la publicidad, Semiótica de la comunicación de masas, Semiótica de la transdiscursividad y Semiótica del discurso audiovisual) presentes en los planes de estudio de Ciencias de la Información en Sevilla. 